\title{
Restriction of Transient Receptor Potential Vanilloid-1 to the Peptidergic Subset of Primary Afferent Neurons Follows Its Developmental Downregulation in Nonpeptidergic Neurons
}

\author{
Daniel J. Cavanaugh, ${ }^{1}$ Alexander T. Chesler, ${ }^{2,3}$ Joao M. Bráz, ${ }^{1}$ Nirao M. Shah, ${ }^{1}$ David Julius, ${ }^{2,3}$ and Allan I. Basbaum ${ }^{1}$ \\ Departments of ${ }^{1}$ Anatomy, ${ }^{2}$ Physiology, and ${ }^{3}$ Cellular and Molecular Pharmacology, University of California, San Francisco, San Francisco, California 94158
}

Primary afferent "pain" fibers (nociceptors) are divided into subclasses based on distinct molecular and anatomical features, and these classes mediate noxious modality-specific contributions to behaviors evoked by painful stimuli. Whether the heat and capsaicin receptor transient receptor potential vanilloid-1 (TRPV1) is expressed heterogeneously across several sensory populations, or is selectively expressed by a unique nociceptor subclass, however, is unclear. Here we used two lines of $\operatorname{Tr} p v 1$ reporter mice to investigate the primary afferent expression of TRPV1, both during development and in the adult. We demonstrate, using Cre-induced lineage tracing, that during development TRPV1 is transiently expressed in a wide range of dorsal root ganglion neurons, and that its expression is gradually refined, such that TRPV1 transcripts become restricted to a specific subset of peptidergic sensory neurons. Finally, the remarkable sensitivity that is characteristic of these reporter mice revealed an innervation of central and peripheral targets by TRPV1+ primary afferents in the adult that is considerably more extensive than has previously been appreciated.

\section{Introduction}

Nociceptors are defined, in part, by their sensitivity to capsaicin, the pungent ingredient in chili peppers. Capsaicin targets transient receptor potential vanilloid-1 (TRPV1), a nonspecific cation channel that is also activated by noxious heat, protons, and membrane-derived lipids such as anandamide (Caterina and Julius, 2001). Consistent with its association with nociceptors, TRPV1 has been localized to small-diameter, unmyelinated $\mathrm{C}$-fibers and medium-diameter, thinly myelinated A $\delta$ fibers in the dorsal root ganglion (DRG) (Tominaga et al., 1998; Michael and Priestley, 1999).

C-fibers are broadly subdivided into two classes based on anatomical and molecular properties. The peptidergic class expresses the neuropeptides calcitonin gene-related peptide (CGRP) and substance $\mathrm{P}$ (SP). Axons of these DRG neurons terminate in the most superficial laminae of the spinal cord dorsal horn (laminae I and outer II). The nonpeptidergic subset, which can be marked by its binding of isolectin B4 (IB4), extends axons that terminate in the inner part of lamina II, ventral to the band of peptidergic fiber innervation. (Basbaum et al., 2009). These subsets also innervate different epidermal layers (Zylka et al., 2005)

\footnotetext{
Received March 14, 2011; revised May 17, 2011; accepted May 19, 2011.

Author contributions: D.J.C., N.M.S., D.J., and A.I.B. designed research; D.J.C., A.T.C., and J.M.B. performed research; N.M.S. contributed unpublished reagents/analytic tools; D.J.C., A.T.C., J.M.B., D.J., and A.I.B. analyzed data; D.J.C. and A.I.B. wrote the paper.

This work was supported by NIH Grants R37NS14627 to A.I.B.; R01NS049488 and DP10D006425 to N.M.S.; and R37NS047723 to D.J.; and by support from the Pell Family Foundation to A.I.B. A.T.C. is supported by an NIH postdoctoral training grant from the UCSF Cardiovascular Research Institute. We thank Cindy Yang for the IRESmycCre construct.

Correspondence should be addressed to Allan I. Basbaum at the above address. E-mail: allan.basbaum@ucsf.edu. DOI:10.1523/JNEUROSCI.1299-11.2011

Copyright $\odot 2011$ the authors $\quad 0270-6474 / 11 / 3110119-09 \$ 15.00 / 0$
}

and likely engage distinct central circuits (Bráz et al., 2005; Braz and Basbaum, 2009).

In addition to their molecular and anatomical differences, these populations subserve distinct pain modalities. Thus, we demonstrated that nonpeptidergic afferents that express the Masrelated G-protein-coupled receptor D (MrgprD) selectively contribute to mechanical, and not heat, pain behaviors. In contrast, the peptidergic fibers that express TRPV1 are essential for behaviors evoked by noxious heat, but are dispensable for mechanically evoked behaviors (Cavanaugh et al., 2009).

Several sensory receptors are selectively expressed by nociceptor subsets. It is unclear, however, to what extent TRPV1 expression follows a subset-specific regulation. In the rat, both peptidergic and nonpeptidergic nociceptors express TRPV1 (Tominaga et al., 1998). In contrast, immunohistochemical analysis in the mouse showed that TRPV1 predominates in the peptidergic subset (Zwick et al., 2002). Electrophysiological analyses, however, suggested that TRPV1 is present in a substantial portion of nonpeptidergic neurons, but apparently at levels below the detection limit of immunohistochemistry (Breese et al., 2005; HjerlingLeffler et al., 2007).

To understand better the primary afferent distribution of TRPV1, we recently generated two lines of knock-in mice (Cavanaugh et al., 2011). In the first line (referred to as TRPV1 ${ }^{\text {Cre }}$ mice), Cre recombinase is expressed under the control of the endogenous TRPV1 promoter. In the second line (TRPV1 ${ }^{\text {PLAP-nlacZ }}$ mice), placental alkaline phosphatase (PLAP) and nuclear lacZ (nlacZ) are expressed under the control of the endogenous TRPV1 promoter. Because Cre is "turned on" in the embryo, when the TRPV1 promoter is first active, these Cre mice can be used to generate a fate map of TRPV1-expressing cells. By contrast, PLAP and nlacZ in TRPV1 $1^{\text {PLAP-nlacZ }}$ mice are limited to 
cells that express TRPV1 at any given time. Using these mice, we demonstrate that, during development TRPV1 is transiently expressed in a wide range of DRG neurons, including both the prospective peptidergic and nonpeptidergic populations. However, in the adult, expression is refined such that TRPV1 expression is largely limited to the peptidergic population.

\section{Materials and Methods}

\section{Mice and injections}

Animal experiments were approved by the Institutional Animal Care and Use Committee and conducted in accordance with the NIH Guide for the Care and Use of Laboratory Animals and the recommendations of the International Association for the Study of Pain. The TRPV1 ${ }^{\text {Cre }}$, TRPV1 ${ }^{\text {PLAP-nlacZ, R26R-lacZ, and R26R-EYFP mice were described pre- }}$ viously (Soriano, 1999; Srinivas et al., 2001; Cavanaugh et al., 2011). For resiniferatoxin (RTX) injections, mice of either sex were anesthetized with $1.5 \%$ isoflurane and injected subcutaneously with escalating doses of RTX on consecutive days (Sándor et al., 2009) (30, 70, 100, and 200 $\mathrm{mg} / \mathrm{ml}$ ), followed by another $200 \mathrm{mg} / \mathrm{ml}$ injection $7 \mathrm{~d}$ later. Histology was performed 7-10 d following the final RTX injection.

\section{PLAP and lac Z detection}

PLAP localization. Mice of either sex were perfused with $10 \mathrm{ml}$ HEPESbuffered saline (HBS) followed by $20 \mathrm{ml}$ of ice-cold 3.7\% formaldehyde. Brain, spinal cord, peripheral tissue, DRG, trigeminal ganglion (TG), and nodose ganglion (NG) were dissected out, postfixed $3-4 \mathrm{~h}$ at $4^{\circ} \mathrm{C}$ and cryoprotected overnight $(\mathrm{O} / \mathrm{N})$ in $30 \%$ sucrose. For brain and spinal cord, $40-80 \mu \mathrm{m}$ sections were cut on a freezing microtome and processed as free-floating sections. For DRGs, TG, NG, and skin, tissue was frozen at $-80^{\circ} \mathrm{C}$ in Tissue-Tek Optimal Cutting Temperature Compound (Sakura Finetek). We cut $14 \mu \mathrm{m}$ cryostat sections, which were processed on slides. Peripheral tissue (skin, cornea, bladder) was processed as a whole mount.

Sections were collected in ice-cold HBS and then rinsed several times with room temperature HBS. Endogenous alkaline phosphatase activity was inactivated by a $60-90 \mathrm{~min}$ incubation in $72^{\circ} \mathrm{C}$ HBS. Following heat inactivation, sections were rinsed in room temperature HBS, washed $3 \times$ 5 min in $\mathrm{B} 1$ buffer $(0.1 \mathrm{M}$ Tris, $\mathrm{pH} 7.5 ; 0.15 \mathrm{M} \mathrm{NaCl})$ and $3 \times 5$ min in $\mathrm{B} 3$ buffer $\left(0.1 \mathrm{~m}\right.$ Tris, pH 9.5; $0.1 \mathrm{M} \mathrm{NaCl} ; 50 \mathrm{~mm} \mathrm{MgCl}_{2}$ ). PLAP was visualized by incubating sections $\mathrm{O} / \mathrm{N}$ in $0.2 \mathrm{mg} / \mathrm{ml} \mathrm{BCIP}$ (Roche) and $1 \mathrm{mg} / \mathrm{ml}$ NBT (Roche) in B3 buffer. The reaction was stopped by washing several times in HBS containing 1 mM EDTA.

Sections were then fixed for at least $1 \mathrm{~h}$ in $3.7 \%$ formaldehyde at $4{ }^{\circ} \mathrm{C}$ and destained with a series of dehydration and rehydration steps in ethanol until the desired signal-to-noise ratio was reached. Sections were rinsed in HBS and then mounted with Fluoromount G (Southern Biotech).

lacZ localization. Tissue for lacZ detection was processed as described above except that postfixation time was limited to $1.5 \mathrm{~h}$, to preserve enzyme activity. Sections were rinsed in solution A (PBS with $2.0 \mathrm{~mm}$ $\left.\mathrm{MgCl}_{2}\right)$, rinsed briefly with prewarmed $\left(37^{\circ} \mathrm{C}\right)$ solution $\mathrm{B}\left(0.1 \mathrm{M} \mathrm{NaPO}_{4}\right.$; $2 \mathrm{mM} \mathrm{MgCl}_{2} ; 0.2 \% \mathrm{NP} 40 ; 0.1 \% \mathrm{Na}$ deoxycholate) and then incubated $1-3 \mathrm{~d}$ in solution $\mathrm{C}$ solution $\mathrm{B}+0.5 \mathrm{M} \mathrm{K}$ ferrocyanide; $0.5 \mathrm{~m} \mathrm{~K}$ ferricyanide; $0.6 \mathrm{mg} / \mathrm{ml} \mathrm{X}$-gal) at $37^{\circ} \mathrm{C}$. The reaction was stopped with HBS containing $1.0 \mathrm{~mm}$ EDTA. Sections were then fixed for at least $1 \mathrm{~h}$ in $3.7 \%$ formaldehyde at $4^{\circ} \mathrm{C}$, rinsed with $\mathrm{HBS}$, and then mounted with Fluoromount G.

For embryonic tissue, embryos were removed and immersion fixed in $3.7 \%$ formaldehyde at $4^{\circ} \mathrm{C}$ for $1 \mathrm{~h}$, then incubated $\mathrm{O} / \mathrm{N}$ in $20 \%$ sucrose in solution A at $4^{\circ} \mathrm{C}$. Embryos were snap frozen at $-80^{\circ} \mathrm{C}$ in OCT, $20 \mu \mathrm{m}$ cryostat sections were cut, and tissue was processed on slides.

\section{Immunohistochemistry}

Sections were processed for immunohistochemistry as described above for PLAP. After a $1 \mathrm{~h}$ incubation in 5\% normal goat serum in PBS with $0.3 \%$ Triton (NGST) to block nonspecific antibody binding, the sections were incubated $\mathrm{O} / \mathrm{N}$ in primary antibody solution diluted in $1 \%$ NGST. The following day, the sections were washed three times with 1\% NGST, then incubated $2 \mathrm{~h}$ in secondary antibody solution (Alexa- 488 or Alexa-
594, diluted 1:700 in 1\% NGST). After washing three times in $0.1 \mathrm{M} \mathrm{PB}$, sections were mounted and coverslipped with Fluoromount G. Primary antisera included the following: guinea pig anti-TRPV1 (1:1000; gift from D. Julius, University of California, San Francisco, San Francisco, CA); rabbit anti- $\beta$ gal (1:10,000; Cappel); rabbit anti-CGRP (1:1000; Peninsula); guinea pig anti-SP (1:8000; gift from J. Maggio, University of Cincinnati, Cincinnati, OH); and mouse anti-N52 (1:1000; Sigma). For IB4 binding, we used biotinylated IB4 (1:500; Vector Laboratories) instead of primary antiserum. For double labeling images with X-gal staining followed by immunohistochemistry, we performed a standard X-gal reaction with $\mathrm{O} / \mathrm{N}$ incubation in solution $\mathrm{C}$, then washed several times with $0.1 \mathrm{M}$ PB before continuing with the immunohistochemistry protocol. The $\mathrm{X}$-gal reaction product was pseudocolored magenta so that these images could be overlayed with fluorescent immunohistochemical images. To quantify labeling, we counted five sections of L4/5 DRG or nodose ganglion in each of three animals. Percentages are presented as mean \pm SE.

\section{DRG calcium imaging}

DRG neurons were isolated from 6-week-old TRPV1 Cre/R26R-EYFP mice of either sex and dissociated with $0.125 \%$ collagenase $\mathrm{P}$ (Boehringer) solution in calcium- and magnesium-free Hank's solution at $37^{\circ} \mathrm{C}$ for $20 \mathrm{~min}$, pelleted, and resuspended in $0.25 \%$ trypsin at $37^{\circ} \mathrm{C}$ for $2 \mathrm{~min}$. Ganglia were triturated gently with a fire-polished Pasteur pipette in culture medium (MEM/EBSS with 10\% fetal bovine serum, vitamins, penicillin-streptomycin, L-glutamine), cultured overnight on poly-Dlysine-coated coverslips, and subjected to ratiometric calcium imaging the next day. Cells were loaded with $10 \mu \mathrm{m}$ Fura-2-AM (Invitrogen) at $22-25^{\circ} \mathrm{C}$ for $60 \mathrm{~min}$ in extracellular Ringer's solution $(140 \mathrm{~mm} \mathrm{NaCl}, 5$ $\mathrm{mm} \mathrm{KCl}, 2 \mathrm{~mm} \mathrm{CaCl}$, 1 mm MgCl2, $10 \mathrm{~mm}$ D-glucose and $10 \mathrm{~mm}$ sodium HEPES, pH 7.4). Cells were illuminated using a xenon light source and filter wheel (Lambda LS and Lambda-10, Sutter Instruments) for $300 \mathrm{~ms}$, alternately at 350 and $380 \mathrm{~nm}$ (bandpass filters from Chroma Technology). Fluorescence emission at $>480 \mathrm{~nm}$ (long-pass filter from Chroma Technology) was captured with an intensified CCD camera (Hamamatsu), and digitized, background corrected, and analyzed with the MetaFluor imaging system (Molecular Devices). Backgroundcorrected 340/380 ratio images were collected every $3 \mathrm{~s}$. Cells were stimulated with 1 and $50 \mu \mathrm{M}$ capsaicin, dissolved in $10 \mu \mathrm{M}$ DMSO, followed by high $\mathrm{K}^{+}$Ringer's solution, imaged for enhanced yellow fluorescent protein (EYFP) fluorescence, then fixed and stained for IB4 binding. Cells were considered IB4+ if their IB4 staining intensity was greater than 2 SDs above the mean of several obviously negative cells in the same well (Breese et al., 2005). All neurons that responded to $\mathrm{HiK}+$ solution were included for analysis.

\section{Results}

\section{TRPV1 ${ }^{\text {Cre }}$ in the DRG}

To investigate TRPV1 expression in the DRG, we crossed TRPV1 ${ }^{\text {Cre }}$ mice with various Cre-dependent reporter lines. When we crossed TRPV1 ${ }^{\text {Cre }}$ mice with the R26R-lacZ reporter line (TRPV1 ${ }^{\mathrm{Cre}} / \mathrm{R} 26 \mathrm{R}-\mathrm{lacZ}$ mice) (Soriano, 1999), in which lacZ is expressed in a Cre-dependent manner, we observed lacZ expression in $49.4 \pm 4 \%$ of adult DRG neurons. Cre recombination occurred at a high frequency in both peptidergic and nonpeptidergic neurons $(88.9 \pm 2.6 \%$ and $73.8 \pm 9.8 \%$ of SP + and IB4 + DRG neurons, respectively), and were also lacZ+ (Fig. $1 B, C$ ). In addition to these unmyelinated C-fiber populations, we also observed that $19.4 \pm 4.7 \%$ of myelinated DRG neurons, identified by immunostaining for the neurofilament NF200 (N52), were lacZ+ (Fig. 1D). Thus, TRPV1 ${ }^{\mathrm{Cre}}$ mice exhibit recombination in a wide range of DRG neurons, including a substantial percentage of both peptidergic and nonpeptidergic C-fibers, and in a subset of myelinated neurons.

We also crossed TRPV1 ${ }^{\text {Cre }}$ mice with R26R-EYFP reporter mice (TRPV1 ${ }^{\text {Cre }} /$ R26R-EYFP mice) (Srinivas et al., 2001), in which Cre recombination results in EYFP expression. This line resulted in stronger axonal labeling, which allowed us to record 

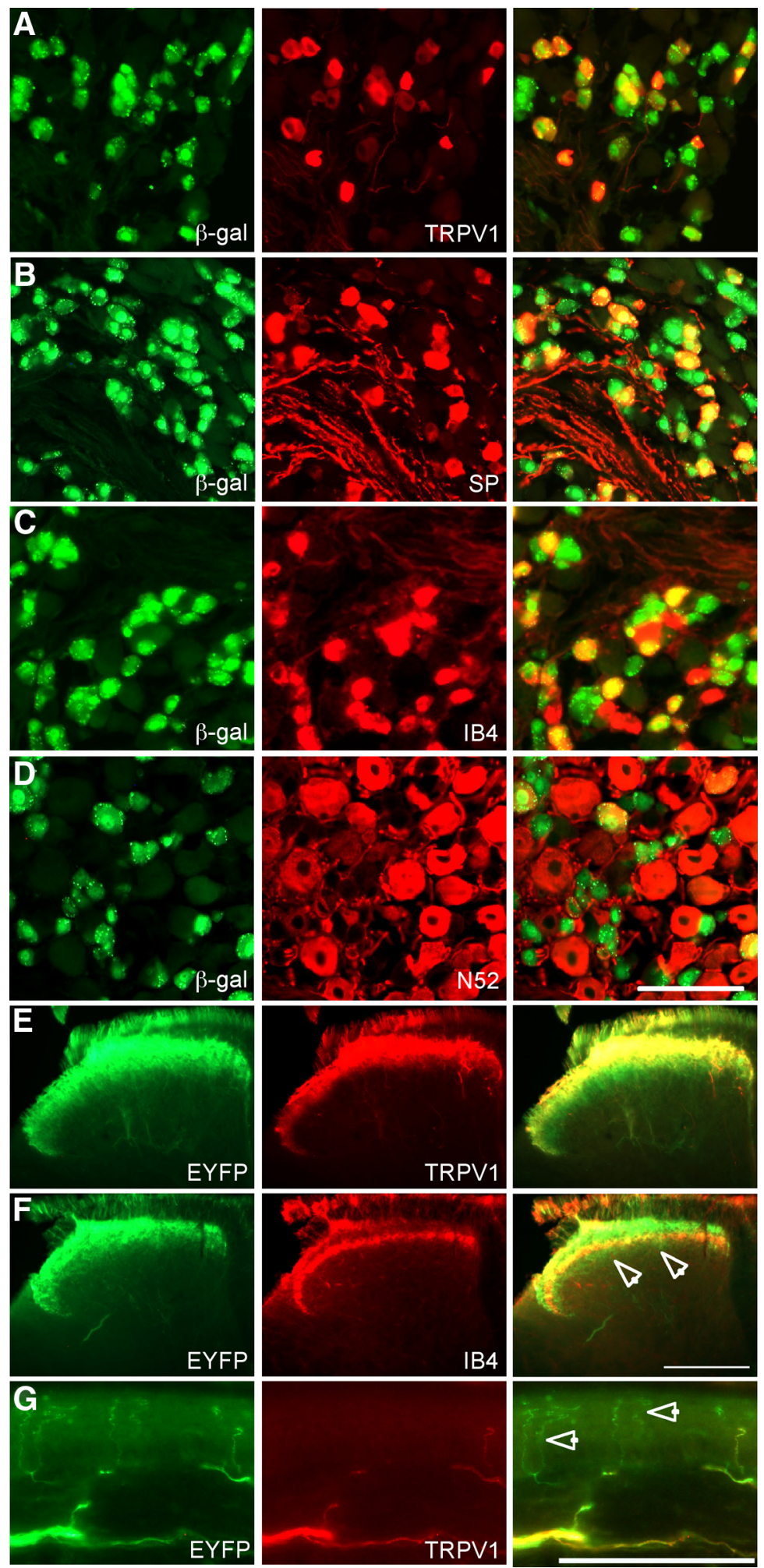

Figure 1. Staining in DRG and spinal cord of TRPV $1{ }^{\mathrm{Cre}}$ mice crossed with Cre-dependent reporter lines. $A-D$, Double labeling of DRG sections from TRPV1 ${ }^{\text {Cre } / R 26 R-l a c Z ~ m i c e ~ f o r ~ l a c Z ~ a n d ~ m a r k e r s ~ o f ~ p r i m a r y ~ a f f e r e n t ~ n e u r o n ~ p o p u l a t i o n s . ~ L e f t ~ p a n e l s ~ a r e ~ s t a i n e d ~}$ for lacZ immunoreactivity (green). Middle panels show the same sections stained by immunohistochemistry for the markers listed (red). Right panels show merged images. $\boldsymbol{E}, \boldsymbol{F}$, Spinal cord sections of TRPV1 ${ }^{\text {Cre }} /$ R26R-EYFP mice are stained for EYFP (left, green) $(\boldsymbol{E})$ and TRPV1 or IB4 (middle, red) $(\boldsymbol{F})$. Merged images are shown on the right. $\boldsymbol{F}$, Arrowheads show EYFP + axons extending ventral to the band of IB4 staining. G, Staining in a section of skin from the hindpaw of a TRPV1 ${ }^{\text {Cre }}$ /R26R-EYFP mouse for EYFP (left, green) and TRPV1 (middle, red). A merged image is shown on the right. Arrowheads show EYFP +/TRPV1-afferent endings. Scale bars: $\boldsymbol{D}, \mathbf{G}, 100 \mu \mathrm{m} ; \boldsymbol{F}, 200 \mu \mathrm{m}$. the central and peripheral projections of DRG neurons that had undergone Credependent recombination. EYFP immunostaining in the spinal cord revealed a dense band of staining that included lamina I and outer II, where peptidergic afferents terminate. The terminal pattern extended into inner lamina II, the layer that is innervated by nonpeptidergic afferents (Fig. $1 E, F$ ). Figure $1 F$, in fact, illustrates that there is substantial overlap between EYFP and IB4 staining in the spinal cord, in agreement with the colocalization of lacZ and IB4 in DRG neurons of TRPV $1{ }^{\text {Cre }} /$ R26R-lacZ mice. And, consistent with Cre expression in myelinated DRG neurons, we observed EYFP + afferents terminating ventral to the band of IB4 staining (Fig. $1 F$, arrowheads) into an area that is targeted by projections from myelinated mechanoreceptive afferents (Neumann et al., 2008).

This pattern of DRG expression and spinal cord termination differs considerably from that reported in the adult mouse, using antibodies directed against TRPV1. The latter studies concluded that TRPV1 is restricted to peptidergic afferents (Zwick et al., 2002). One possibility is that the discrepancy resulted from the remarkable sensitivity of the TRPV $1{ }^{\text {Cre }}$ line compared with traditional immunohistochemical techniques for the detection of TRPV1 expression. On the other hand, as the TRPV $1{ }^{\text {Cre }}$ line generates a fate map of TRPV1 expression, we hypothesized that the lacZ expression in IB4-binding DRG neurons of TRPV 1 Cre/R26R-lacZ mice in fact represented cells that expressed TRPV1 at some point during development, but not in the adult. Consistent with this latter possibility, when we compared the fate map of TRPV1, as represented by lacZ staining, and the adult expression pattern revealed by TRPV1 immunoreactivity, we found that only $50.8 \pm 2.5 \%$ of lac $Z+$ neurons were TRPV1+ (Fig. 1A).

A comparison of TRPV1 and EYFP staining in the spinal cord of TRPV 1 Cre/ R26R-EYFP mice also revealed that EYFP+ afferents extended into deeper laminae of the spinal cord dorsal horn (Fig. 1E). Finally, EYFP + afferents in sections of paw skin exhibited ramified endings that extended into the most superficial layers of the epidermis, in a manner and density similar to those of nonpeptidergic afferents (Zylka et al., 2005). Importantly, when studied in the adult, these axons generally did not colocalize with TRPV1 immunoreactivity (Fig. 1G). Together, these data suggest that TRPV1 expression in the mouse is developmentally regulated such that the distribu- 
A

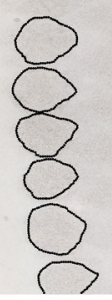

E11.5 B

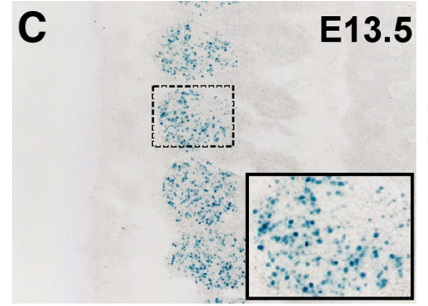

E

C
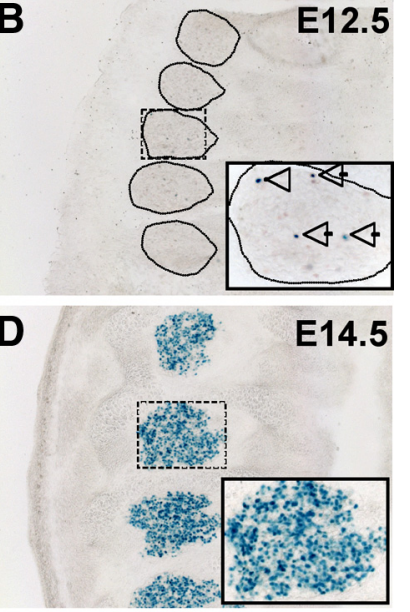

E16.5
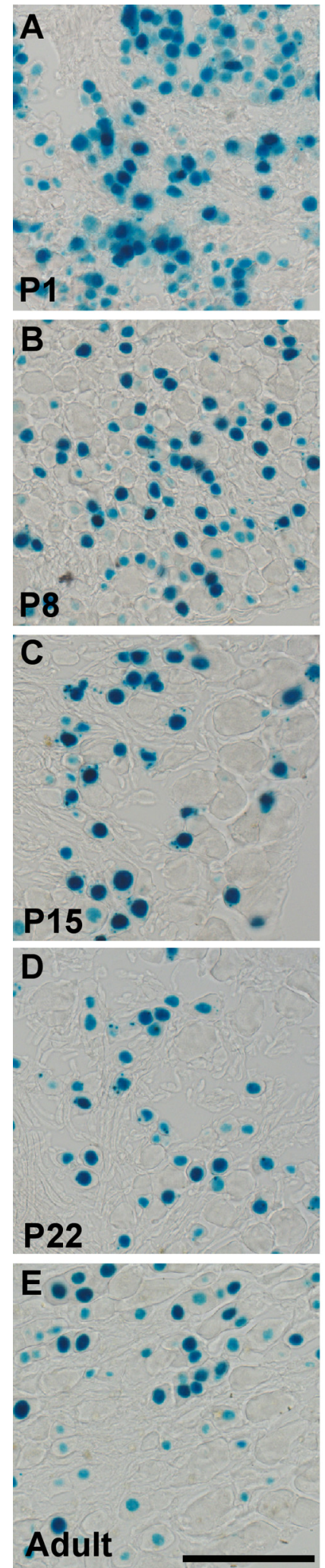
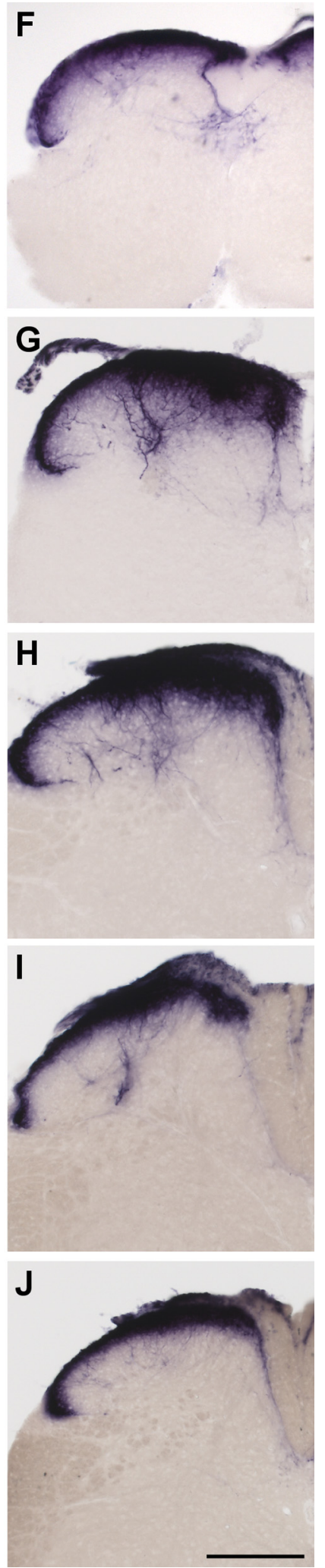

Figure 3. Postnatal regulation of TRPV1 in DRG neurons. $\boldsymbol{A}-\boldsymbol{E}$, nlacZ staining in DRG sections of TRPV1 ${ }^{\text {PLAP-nlacZ }}$ mice. $A, A t P 1,59.7 \pm 1.7 \%$ of DRG neurons were nlacZ $+. B, A t P 8,45.4 \pm$ $4.4 \%$ of DRG neurons were nlacZ+. $\boldsymbol{C}-\boldsymbol{E}, \mathrm{By} P 15,33.6 \pm 4.4 \%$ of neurons were nlacZ $+(\boldsymbol{C})$, and this percentage stayed constant at P22 (D), and in the adult mouse $(\boldsymbol{E}) . \boldsymbol{F}-\boldsymbol{J}$, PLAP staining of spinal cord sections of TRPV1 ${ }^{\text {PLAP-nlacz }}$ mice at P1 $(\boldsymbol{F})$, P8 $(\boldsymbol{G}), \mathrm{P} 15(\boldsymbol{H}),(\boldsymbol{I})$ P22, and adult $(\boldsymbol{J})$. Note that staining becomes progressively restricted to the outermost dorsal horn laminae. The percentage of nlacZ + DRG neurons at each time point is given as mean \pm SEM. Scale bars: $\boldsymbol{E}$, $100 \mu \mathrm{m} ; J, 200 \mu \mathrm{m}$. 

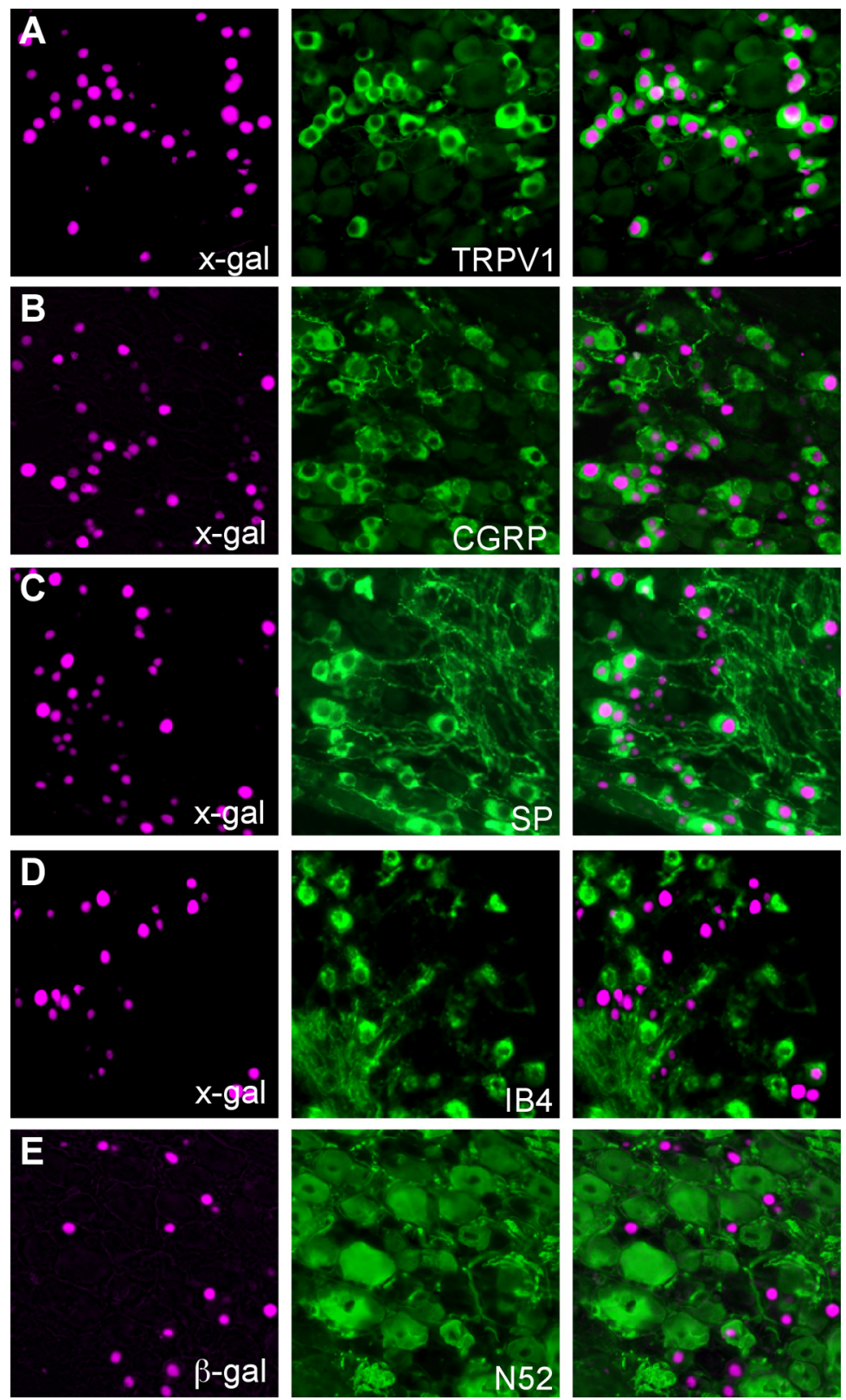

Figure 4. Adult expression of TRPV1. $\boldsymbol{A}-\boldsymbol{E}$, Double labeling of DRG sections from TRPV1 ${ }^{\text {PLAP-nlacZ }}$ mice for nlacZ and markers of primary afferent neuron populations. $\boldsymbol{A}-\boldsymbol{E}$, Left panels show nlacZ staining with the X-gal reaction (pseudocolored magenta). Middle panels show the same sections stained by immunohistochemistry for the markers listed. Right panels show merged images. dergic C-fibers. Thus, $64.0 \pm 2.3 \%$ of nlacZ+ DRG neurons colabeled for CGRP (Fig. 4B). Furthermore, $82.4 \pm$ $1.3 \%$ of SP+ cells and $48.9 \pm 1.9 \%$ of CGRP+ cells were nlacZ+ (Fig. 4B,C). As SP primarily marks unmyelinated peptidergic nociceptors, while CGRP is expressed in both myelinated and unmyelinated peptidergic afferents, these results suggest that TRPV1+ cells in the adult account for nearly all of the unmyelinated, peptidergic subtype. In contrast, only $11.5 \pm 0.7 \%$ of nlacZ+ DRG neurons bound IB4 (Fig. 4D). This number is greater than that obtained by immunohistochemical analyses (Zwick et al., 2002), presumably because of the greater sensitivity of nlacZ staining. Finally, we found that only a very small percentage of nlac $Z+$ neurons $(4.7 \pm 2.2 \%)$ expressed the neurofilament NF200 (Fig. 4E). Interestingly, given the percentage of nlacZ+ neurons that colabels with these various markers, it is clear that a significant number $(\sim 20 \%)$ of TRPV1+ neurons are negative for both peptidergic and nonpeptidergic markers, which indicates that the peptidergic and nonpeptidergic classes of C-fiber do not account for the entirety of unmyelinated primary afferent neurons.

\section{TRPV1 in the TG}

The TGs contain the cell bodies of sensory neurons that innervate the head and neck, including several unique targets [e.g., teeth (Fehrenbacher et al., 2009), cornea (Bates et al., 2010), and cranial dura (Dux et al., 2009)]. Because of these specialized targets, it is possible that TG nociceptors differ from DRG neurons in their general neurochemical makeup. We therefore assayed TRPV1 expression in trigeminal afferents using the TRPV1 ${ }^{\text {PLAP-nlacZ }}$ mice. Somewhat surprisingly, TRPV1 was relatively less abundant in the TG compared with the DRG; only $20.3 \pm 1.0 \%$ of adult TG neurons were nlacZ + . Nevertheless, weeks. This enhanced developmental expression is manifested as an increase in the percentage of TRPV1+ DRG neurons, and in an expanded termination zone of TRPV1+ afferents in the spinal cord.

\section{Adult expression of TRPV 1 in primary afferent neurons}

We investigated the adult distribution of TRPV1 by performing nlacZ staining in TRPV1 ${ }^{\text {PLAP-nlacZ }}$ mice, followed by immunohistochemistry for various markers of nociceptor populations. Compared with TRPV1 ${ }^{\text {Cre }} / \mathrm{R} 26 \mathrm{R}-\mathrm{lacZ}$ mice, in which only half of the DRG neurons marked by reporter molecules could be labeled with antibodies directed against TRPV1, virtually all (95 $\pm .4 \%)$ of nlacZ+ DRG neurons in TRPV1 ${ }^{\text {PLAP-nlacZ }}$ mice were TRPV1 immunoreactive (Fig. 4A). Corroborating previous reports, and in contrast to the broad distribution observed in TRPV1 ${ }^{\mathrm{Cre}}$ mice, we found that TRPV1 in the adult is enriched selectively in pepti- as in the DRG, TRPV1 in the TG was preferentially expressed in peptidergic neurons. Thus, $73.2 \pm 12.0 \%$ of nlacZ + neurons in the TGs were CGRP +, while $15.1 \pm 1.8 \%$ of nlacZ+ bound IB4

\section{Innervation of peripheral and central tissue by TRPV1+ afferents}

To investigate the innervation of peripheral and central tissues by TRPV1+ afferents in the adult, we stained these tissues for PLAP in TRPV $1^{\text {PLAP-nlacZ }}$ mice. We observed extensive axonal labeling of peripheral tissues, including the bladder (Fig. $5 \mathrm{~A}$ ), cornea (Fig. $5 B$ ), and glabrous and hairy skin of the hindpaw (Fig. 5C). TRPV1 fibers in glabrous skin of the paw generally stratified parallel to the epidermal/dermal boundary (Fig. $5 E$, arrows) and sent regular projections into the innermost epidermal layers (Fig. 5E, arrowheads). Occasionally, we observed axonal endings that ex- 

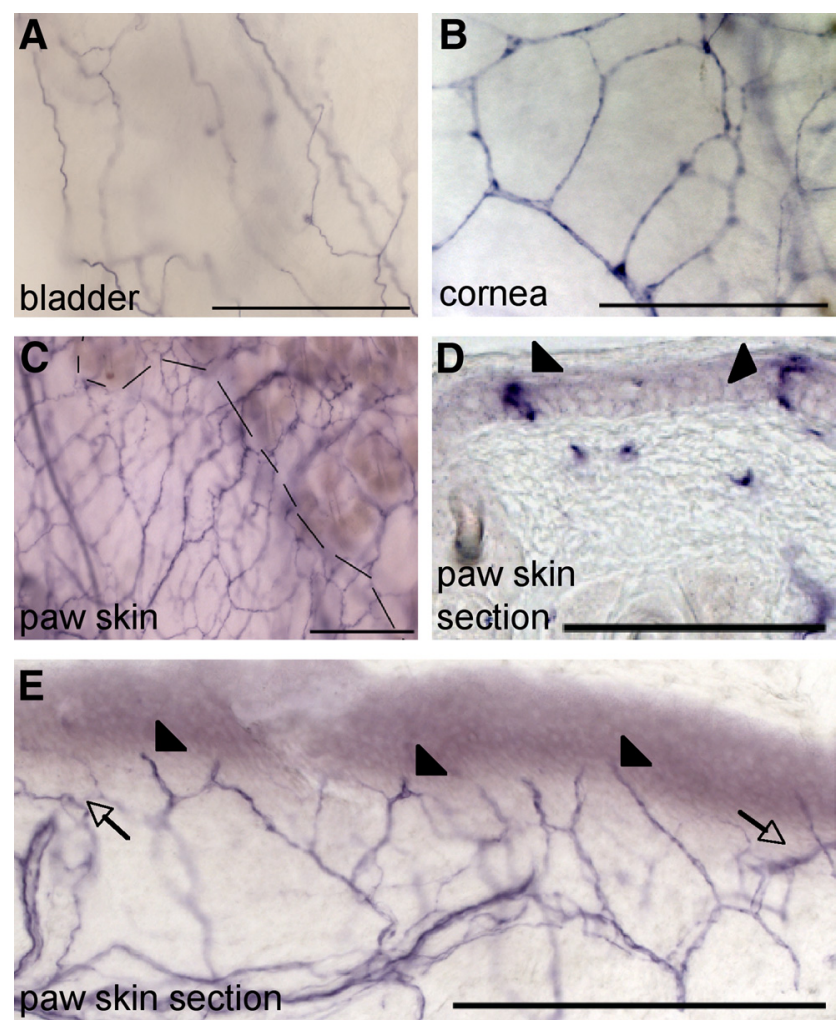

Figure 5. PLAP staining in peripheral tissues targeted by DRG neurons. $A$, PLAP staining in whole-mount bladder. $\boldsymbol{B}$, PLAP staining in whole-mount cornea. C, PLAP staining in wholemount paw skin. Dashed line shows boundary between hairy (right) and glabrous (left) skin. $\boldsymbol{D}$, In $14 \mu \mathrm{m}$ sections of paw skin, we occasionally observed PLAP + fibers extending into the outermost layer of the epidermis (arrowheads). $\boldsymbol{E}$, PLAP + afferent fibers were generally observed running parallel to the dermal/epidermal border (arrows) and regularly extended into the innermost epidermal layers (arrowheads). Scale bars: $A-C, 200 \mu \mathrm{m} ; \boldsymbol{D}, \boldsymbol{E}, 100 \mu \mathrm{m}$.

tended more superficially, at times contacting the outermost epidermal layer (Fig. 5D, arrowheads).

In addition to the spinal cord (Fig. 3J), PLAP prominently labeled other CNS regions that receive direct inputs from primary afferent nociceptors. For example, we observed dense PLAP axonal staining in the superficial layers of the nucleus caudalis (Fig. $6 \mathrm{~A}$, arrow), which is targeted by TG afferents, as well as in the nucleus of the solitary tract (NTS) (Fig. 6A, arrowhead) and in the area postrema of the medulla, both of which are innervated by vagal afferents originating in the nodose ganglion. Consistent with expression in the NTS, we noted PLAP staining of the solitary tract (Fig. $6 \mathrm{~B}$, arrow) and nlacZ expression in the nodose ganglion (data not shown). Interestingly, the overlap of IB4 binding with nlacZ in the NG was significantly greater than in the DRG $(38.2 \pm 3.2 \%$ of nodose nlacZ+ cells were IB4+).

The great sensitivity of the PLAP detection method is well illustrated by our observation of extended trigeminal afferent terminal fields in the brainstem. For example, we found PLAP expression in axons extending from the ventromedial border of nucleus caudalis, which receives nociceptor input from the face, into the region of the rostral ventrolateral reticular nucleus (Fig. $6 C$ ), raising the possibility of a monosynaptic input from TRPV1positive afferents to motor neurons of the nucleus ambiguus. Strikingly, we also observed PLAP + axons projecting throughout the dorsolateral pontine parabrachial nucleus (Fig. 6E, F, arrows), with especially dense terminations in the CGRP + cell body-rich, external lateral parabrachial nucleus (Fig. 6E, arrow). This region has previously been implicated in the polysynaptic
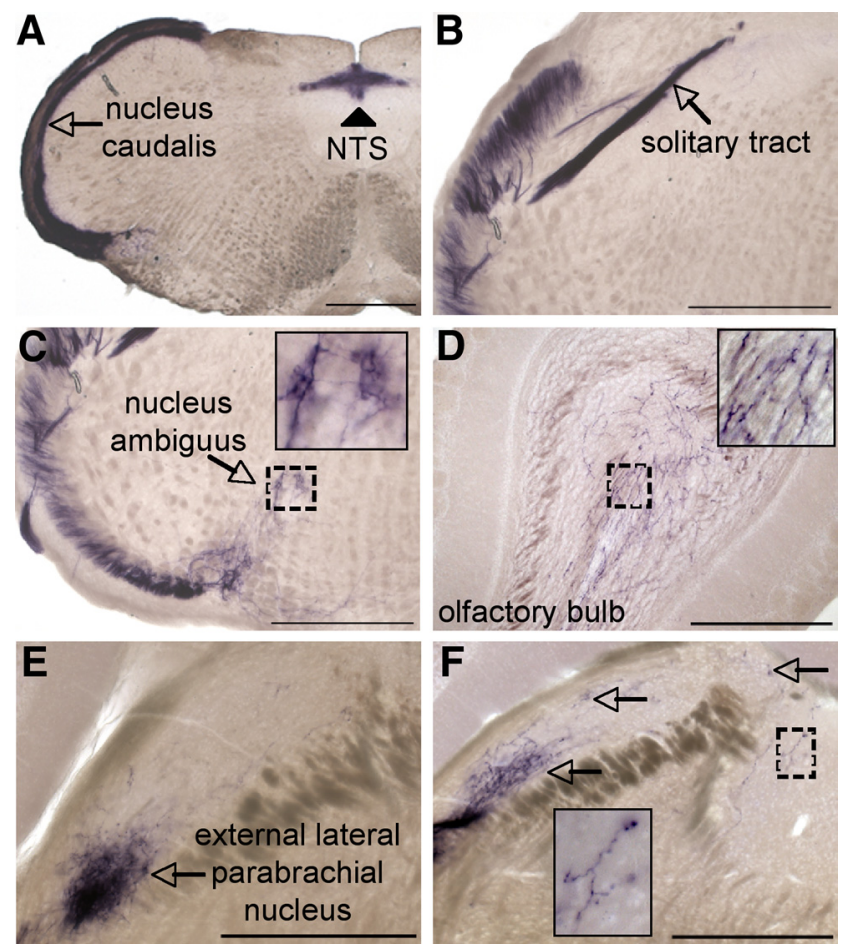

Figure 6. PLAP staining in central tissues targeted by DRG neurons. $\boldsymbol{A}$, PLAP staining in nucleus caudalis (arrow) and the nucleus of the solitary tract (arrowhead) of the medulla. $\boldsymbol{B}$ Robust PLAP staining in the solitary tract (arrow). C, PLAP staining in the ventral medulla (inset shows magnification of boxed area, near nucleus ambiguus). $\boldsymbol{D}$, PLAP + axons in the granule layer of the olfactory bulb (inset shows magnification of boxed area). $\boldsymbol{E}$, External lateral parabrachial nucleus receives extensive PLAP+ innervation (arrow). $\boldsymbol{F}$, PLAP + fibers extend throughout the parabrachial nucleus (arrows). Insets show magnification of boxed areas. Scale bars, $500 \mu \mathrm{m}$.

transmission of nociceptive inputs to the amygdala. Finally, we found extensive axonal PLAP staining in the granule layer of the olfactory bulb (Fig. $6 D$ ), as well as sparser innervation of the glomerular region of the bulb. Importantly, PLAP labeling was completely eliminated following systemic administration of the neurotoxic TRPV1 agonist RTX (Fig. 7), indicating that the anatomical expression patterns represent functional TRPV1 channels. That axonal PLAP labeling in the brain derives from primary afferent origin is suggested by the fact that we do not observe PLAP + cell bodies in the brain, and that none of these brain areas are labeled by nlacZ in TRPV1 ${ }^{\text {PLAP-nlacZ }}$ mice (Cavanaugh et al., 2011).

\section{Calcium imaging in DRG neurons of TRPV1 $^{\mathrm{Cre}} / \mathrm{R} 26 \mathrm{R}$-EYFP mice}

The more restricted pattern of expression in the TRPV1 ${ }^{\text {PLAP-nlacZ }}$ mice, compared with that observed in the TRPV1 ${ }^{\mathrm{Cre}}$ line, suggested that TRPV1 is extinguished by both nonpeptidergic and myelinated DRG neurons during development, resulting in a loss of functional TRPV1 expression in these populations. It is, of course, also possible that these subsets of neurons express functional TRPV1 in the adult mouse, but at levels below the detection limit of immunohistochemical staining, or of X-gal staining in TRPV1 $1^{\text {PLAP-nlacZ }}$ mice. To differentiate between these two possibilities, we next performed calcium imaging experiments in TRPV1 ${ }^{\text {Cre }}$ /R26R-EYFP mice and monitored responses to the potent and selective TRPV1 agonist capsaicin (Fig. 8).

We reasoned that if TRPV1 expression is extinguished by subsets of DRG neurons during development, this would be reflected 


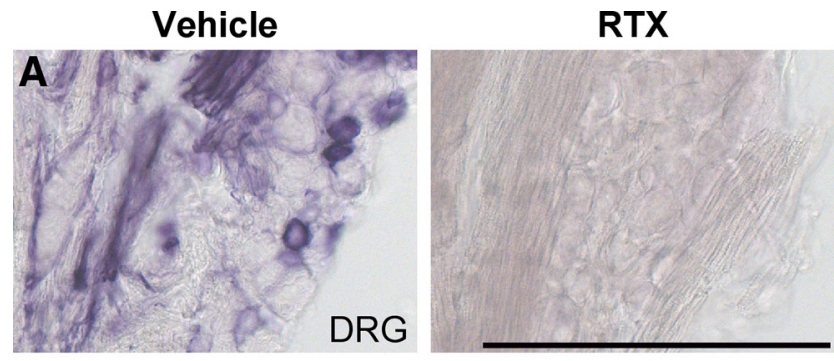

B
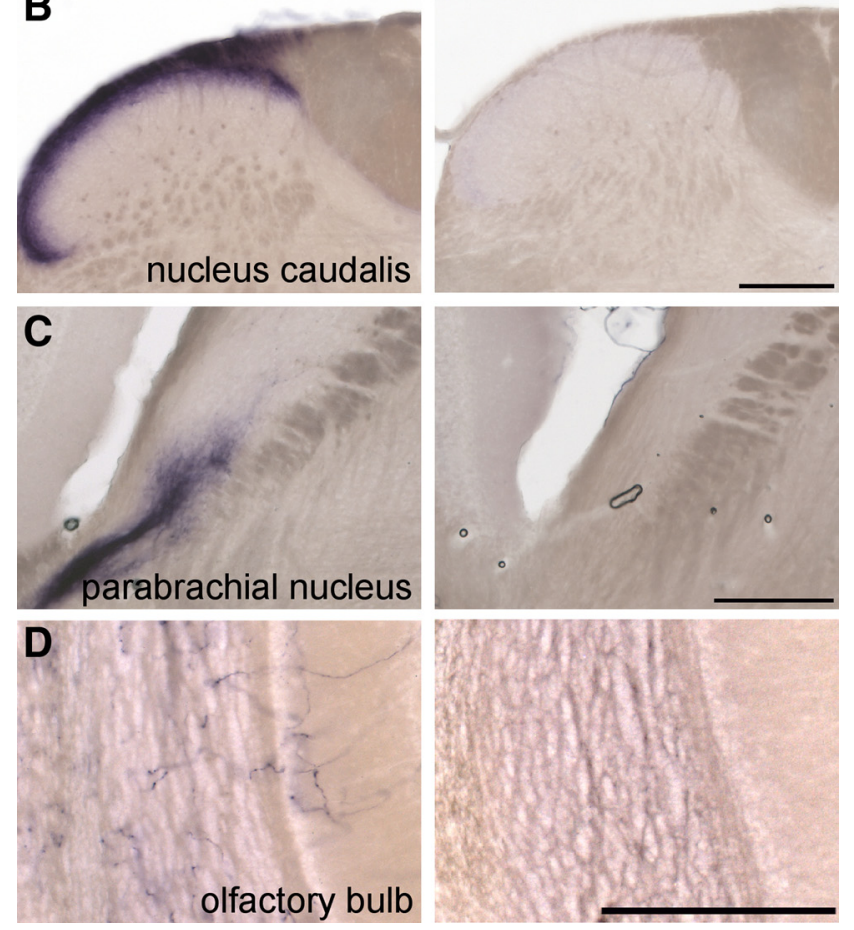

Figure 7. Systemic RTX injection elminates PLAP staining in spinal cord and brain. $\boldsymbol{A}-\boldsymbol{D}$, PLAP staining in DRG $(\boldsymbol{A})$, nucleus caudalis $(\boldsymbol{B})$, parabrachial nucleus $(\boldsymbol{C})$, and olfactory bulb $(\boldsymbol{D})$ is eliminated following RTX injection. Left panels show PLAP staining in vehicle-injected control mice, and right panels show equivalent areas in RTX-injected mice. Scale bars, $200 \mu \mathrm{m}$.

by EYFP + neurons in culture that do not respond to capsaicin. The great majority (93\%) of capsaicin-responsive neurons in TRPV 1 Cre/R26R-EYFP mice were EYFP+, indicating that most TRPV1-expressing cells in the adult had undergone Credependent recombination. However, consistent with a developmental downregulation of TRPV1 in DRG neurons, we found that only $64 \%(76 / 119)$ of EYFP + neurons in the adult responded to application of capsaicin. By contrast, we have previously shown that $>95 \%$ of adult nlacZ + DRG neurons cultured from TRPV1 ${ }^{\text {PLAP-nlacZ }}$ mice are capsaicin responsive (Cavanaugh et al., 2011). We further examined the neurochemical phenotype of those EYFP + cells that did not respond to capsaicin, and found that $56 \%$ bound the lectin IB4. Furthermore, although nearly $89 \%$ of IB $4+$ cells in culture were EYFP + , only $31 \%$ of IB $4+$ cells were capsaicin responsive. Finally, only $\sim 20 \%$ of capsaicinresponsive cells were $\mathrm{IB} 4+$, in general agreement with our finding that $12 \%$ of nlacZ + DRG neurons in adult TRPV $1{ }^{\text {PLAP-nlacZ }}$ mice were IB4 + . In total, these data definitively demonstrate that TRPV1 is developmentally expressed more broadly than it is in the adult, and that a substantial percentage of IB4-binding, nonpeptidergic neurons lose functional TRPV1 expression during development.

\section{Discussion}

Extensive innervation of peripheral and CNS tissue by TRPV1+ afferents

In the present study, we used a genetic approach to document the primary afferent distribution of TRPV1 in the developing and adult mouse. This approach has several advantages compared with traditional techniques used to localize TRPV1, including enhanced sensitivity and much greater signal to background. Analysis of knock-in mice in which we expressed reporter molecules under the control of the endogenous TRPV1 promoter demonstrated that, in the adult mouse, TRPV1 is largely restricted to the peptidergic population of DRG neurons. In addition, the excellent sensitivity of PLAP staining in adult TRPV $1^{\text {PLAP-nlacZ }}$ mice allowed for a greater appreciation of the extent of primary afferent innervation in both central and peripheral tissues. The greater signal-to-noise ratio and minimal background using PLAP is particularly advantageous compared with studies using TRPV1 antisera. For example, while others reported barely detectable levels of TRPV1 protein in the skin under basal conditions (Ji et al., 2002; Elitt et al., 2006), we observed dense axonal arbors coursing throughout the skin. We also observed a much denser corneal innervation than did an earlier immunohistochemical analysis (Murata and Masuko, 2006), and also found extensive PLAP labeling in the olfactory bulb. Although the olfactory bulb is an established target of peptidergic trigeminal afferents (Finger and Böttger, 1993), the extent of innervation that we detected greatly exceeds what was previously reported, especially with regard to innervation of the granule layer of the bulb.

We also observed prominent PLAP labeling in axons that coursed throughout the parabrachial nucleus. As the only PLAP + cell bodies in the nervous system of TRPV $1^{\text {PLAP-nlacZ }}$ mice are in sensory ganglia (Cavanaugh et el., 2011), we hypothesize that the parabrachial staining is of primary afferent origin. It follows that neurons in this region receive significant direct (i.e., monosynaptic) primary afferent nociceptive input. It is of particular interest that the densest PLAP staining is in the in the external lateral portion of the parabrachial nucleus, a region that receives polysynaptic nociceptive input from nociresponsive lamina I neurons of the dorsal horn and trigeminal nucleus caudalis (Feil and Herbert, 1995; Jasmin et al., 1997). These parabrachial neurons in turn project to the amygdala, which has been implicated in the emotional quality of the pain experience. Direct access of trigeminal primary afferent neurons to the parabrachial nucleus would provide an alternative circuit that could contribute to this aspect of the pain experience, in addition to the spino(trigemino-)parabrachial pathway. It is also possible that these TRPV1 + fibers contact parabrachial areas involved in descending pain modulation, such as the Kölliker-Fuse nucleus (Willis and Westlund, 1997). Interestingly, there does not appear to be a DRG homolog of the direct trigeminal afferent input to the parabrachial nucleus, perhaps because the input relates to the structures uniquely innervated by TG nociceptors. Studies that determine the peripheral target of the TG ganglion cells that project to the PB would therefore be informative.

\section{Developmental downregulation of TRPV1 expression in nociceptors}

By monitoring the developmental time course, we found that TRPV1 expression emerges in a very small number of DRG neurons at E12.5. Over the next 2 embryonic days, the number of TRPV1+ cells greatly increases, after which, during late embryonic and early postnatal development, there is a gradual decline in the TRPV1+ 
population. These findings are in agreement with functional imaging studies, which assayed capsaicin responsiveness of cultured DRG neurons throughout development, and found that the percentage of capsaicinresponsive neurons decreases over time (Hjerling-Leffler et al., 2007).

Without a means to prospectively mark early TRPV $1+$ cells, however, it was previously not possible to determine whether the developmental reduction in the percentage of TRPV1+ DRG neurons was due to a downregulation of TRPV1 in a subset of DRG neurons, or to the wave of cell death that occurs in the DRG during this time period (Coggeshall et al., 1994). In fact, we found that the reporter molecule expression in TRPV $1^{\text {Cre }} / \mathrm{R} 26 \mathrm{R}-\mathrm{lacZ}$ and TRPV 1 Cre/R26R-EYFP mice persists into adulthood, even in cells in which TRPV1 is no longer expressed. This Creinduced lineage analysis allowed us to follow the fate of cells that express TRPV1 embryonically. Our analysis showed that almost half of the lacZ+ cells in adult TRPV1 ${ }^{\text {Cre} / R 26 R-l a c Z ~ m i c e ~ w e r e ~ n o t ~ T R P V 1 ~}$ immunoreactive, and this included a very high percentage of the nonpeptidergic population of unmyelinated DRG neurons (Zwick et al., 2002). These observations are highly consistent with those derived from an analysis of bacteria artificial chromosome (BAC) transgenic mice in which Cre was expressed under the control of the TRPV1 gene (Mishra et al., 2011) and suggest that there is a developmental downregulation of TRPV1 in some DRG neurons. However, histochemical analysis alone of TRPV $1{ }^{\text {Cre }}$ mice, whether based on a BAC or knock-in transgenic, cannot definitively determine whether the discrepancy between the percentage of DRG neurons that have undergone Cre recombination, compared with those that are TRPV1 immunoreactive, reflects a developmental loss of TRPV1, or whether it is due to lower sensitivity of TRPV1 antisera compared with genetic marking strategies. This is especially true as it has been argued that TRPV1 antibodies underestimate the number of TRPV1+ cells in the DRG (Breese et al., 2005; Hjerling-Leffler et al., 2007).

By using two independent transgenic lines combined with functional imaging, however, we established that the developmental reduction of TRPV1 in the DRG is, in fact, a result of selective loss of TRPV1 from specific primary afferent subsets. First, we observed that nlacZ in adult TRPV1 ${ }^{\text {PLAP-nlacZ }}$ mice showed a preferential distribution in the peptidergic population. Since reporter molecule expression in this line is driven by active transcription at the Trpv1 locus, nlacZ is only observed in cells in which TRPV1 is expressed at the time of analysis. We have previously demonstrated the greater sensitivity of these mice compared with traditional histochemical techniques (Cavanaugh et al., 2011). Perhaps more convincing, calcium-imaging experiments in TRPV1 ${ }^{\text {Cre } / R 26 R-E Y F P ~ m i c e ~ c l e a r l y ~ s h o w e d ~ t h a t ~ m a n y ~}$ EYFP + cells were not responsive to capsaicin. Furthermore, a large percentage of these EYFP+ cells that did not respond to capsaicin showed a nonpeptidergic phenotype. Based on these
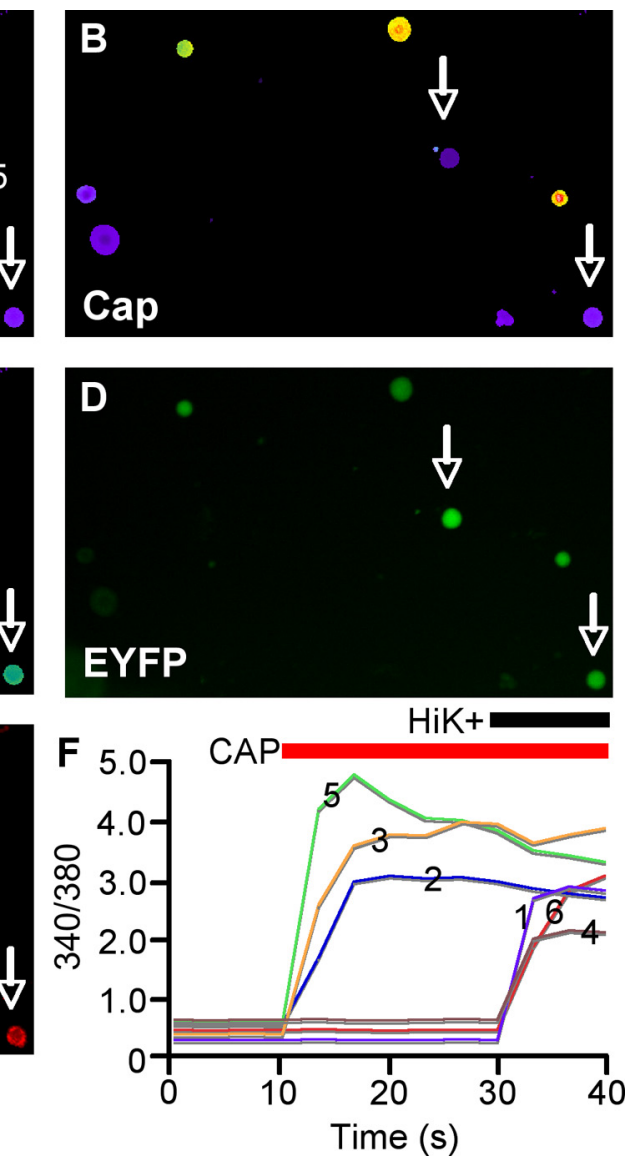

Figure 8. Calcium imaging of capsaicin responsiveness in cultured TRPV1 ${ }^{\text {Cre }} / R 26 R-E Y F P$ DRG neurons. $\boldsymbol{A}-\boldsymbol{C}$, Cultured DRG neurons from 6-week-old mice were imaged with Fura-2-AM dye at background $(\boldsymbol{A})$, and following stimulation with 1 or $50 \mu \mathrm{M}$ capsaicin $(\boldsymbol{B})$, and high-K ${ }^{+}$Ringer's solution (C).D, Endogenous EYFP signal from cells recorded in $\boldsymbol{A}-\boldsymbol{C}$. $\boldsymbol{E}$, Cells were then fixed and assayed for IB4 binding. $\boldsymbol{E}, 340 / 380$ ratios of cells as numbered in $\boldsymbol{A}$. Arrows point to EYFP+, IB4-binding cells that were unresponsive to capsaicin. Scale bar, $100 \mu \mathrm{m}$.

results, we conclude that a large population of DRG neurons that express TRPV1 transiently during development does not die. Rather, these neurons persist into adulthood and largely constitute the TRPV1-negative population of unmyelinated, nonpeptidergic nociceptors.

It is unclear what mediates the downregulation, but the timing of this developmental regulation coincides with the emergence of other characteristics of nonpeptidergic neurons, including the transition from TrkA to c-Ret expression, and the onset of IB4 binding (Molliver et al., 1997; Chen et al., 2006; Hjerling-Leffler et al., 2007). As we have demonstrated here, the TRPV1+ population comprises a large percentage of the so-called peptidergic class of nociceptors. However, $\sim 35 \%$ of TRPV1 + neurons do not express neuropeptides, and an appreciable population $(\sim 12$ $20 \%$ ) of TRPV1+ neurons (whether determined by reporter molecule expression in knock-in mice, or by capsaicin responses in cultured DRG neurons) bind the lectin IB4. We and others have recently shown that pharmacological ablation of TRPV1+ neurons in the adult results in selective and complete deficits in behavioral responses to noxious heat (Cavanaugh et al., 2009, Mishra and Hoon, 2010). It therefore appears that, while it may be heuristically useful to classify nociceptors based on traditional markers of peptidergic and nonpeptidergic neurons, it is the presence or absence of TRPV1 that divides nociceptors into functionally relevant populations. This assertion is supported by the fact that chemical-genetic elimination of DRG neurons that ex- 
press the MrgprD receptor, which comprise the vast majority of TRPV1-/IB4+ neurons, results in selective noxious mechanical deficits (Cavanaugh et al., 2009). Importantly, TRPV1+ and TRPV1 - afferents target distinct spinal cord laminae in adult mice. We have shown that this termination pattern is a result of the downregulation of TRPV1 in certain DRG neurons. The developmental restriction of TRPV1 could thus serve to provide an anatomical substrate for the segregated processing of different noxious modalities in the peripheral and CNS.

Finally, our results offer insight into the development of sensitized pain states caused by early environmental stressors, such as inflammation. Neonatal inflammation can lead to a permanent sensitization of the pain system and a persistent expansion of the termination zone of small-diameter nociceptors in the dorsal horn (Ruda et al., 2000). Our results suggest that this sensitized pain state may arise, in part, from a developmental failure to downregulate TRPV1 expression in the DRG. The pathological persistence of TRPV1 in nonpeptidergic neurons would increase the overall response to TRPV1 agonists and, perhaps more importantly, would expand the population of nociceptors activated by such stimuli, such that circuits normally engaged only by mechanical stimuli could also be activated by noxious heat.

\section{References}

Basbaum AI, Bautista DM, Scherrer G, Julius D (2009) Cellular and molecular mechanisms of pain. Cell 139:267-284.

Bates BD, Mitchell K, Keller JM, Chan CC, Swaim WD, Yaskovich R, Mannes AJ, Iadarola MJ (2010) Prolonged analgesic response of cornea to topical resiniferatoxin, a potent TRPV1 agonist. Pain 149:522-528.

Bráz JM, Basbaum AI (2009) Triggering genetically-expressed transneuronal tracers by peripheral axotomy reveals convergent and segregated sensory neuron-spinal cord connectivity. Neuroscience 163:1220-1232.

Braz JM, Nassar MA, Wood JN, Basbaum AI (2005) Parallel "pain" pathways arise from subpopulations of primary afferent nociceptor. Neuron 47:787-793.

Breese NM, George AC, Pauers LE, Stucky CL (2005) Peripheral inflammation selectively increases TRPV1 function in IB4-positive sensory neurons from adult mouse. Pain 115:37-49.

Caterina MJ, Julius D (2001) The vanilloid receptor: a molecular gateway to the pain pathway. Annu Rev Neurosci 24:487-517.

Cavanaugh DJ, Lee H, Lo L, Shields SD, Zylka MJ, Basbaum AI, Anderson DJ (2009) Distinct subsets of unmyelinated primary sensory fibers mediate behavioral responses to noxious thermal and mechanical stimuli. Proc Natl Acad Sci U S A 106:9075-9080.

Cavanaugh DJ, Chesler AT, Jackson AC, Sigal YM, Yamanaka H, Grant R, O’Donnell D, Nicoll RA, Shah NM, Julius D, Basbaum AI (2011) Trpv1 reporter mice reveal highly restricted brain distribution and functional expression in arteriolar smooth muscle cells. J Neurosci 31:5067-5077.

Chen CL, Broom DC, Liu Y, de Nooij JC, Li Z, Cen C, Samad OA, Jessell TM, Woolf CJ, Ma Q (2006) Runx1 determines nociceptive sensory neuron phenotype and is required for thermal and neuropathic pain. Neuron 49:365-377.

Coggeshall RE, Pover CM, Fitzgerald M (1994) Dorsal root ganglion cell death and surviving cell numbers in relation to the development of sensory innervation in the rat hindlimb. Brain Res Dev Brain Res 82:193-212.

Dux M, Rosta J, Sántha P, Jancsó G (2009) Involvement of capsaicinsensitive afferent nerves in the protenase-activated receptor 2-mediated vasodilatation in the rat dura mater. Neuroscience 161:887-894.

Elitt CM, McIlwrath SL, Lawson JJ, Malin SA, Molliver DC, Cornuet PK, Koerber HR, Davis BM, Albers KM (2006) Artemin overexpression in skin enhances expression of TRPV1 and TRPA1 in cutaneous sensory neurons and leads to behavioral sensitivity to heat and cold. J Neurosci 26:8578-8587.

Fehrenbacher JC, Sun XX, Locke EE, Henry MA, Hargreaves KM (2009) Capsaicin-evoked iCGRP release from human dental pulp: a model system for the study of peripheral neuropeptide secretion in normal healthy tissue. Pain 144:253-261.

Feil K, Herbert H (1995) Topographic organization of spinal and trigeminal somatosensory pathways to the rat parabrachial and Kolliker-Fuse nuclei. J Comp Neurol 353:506-528.

Finger TE, Böttger B (1993) Peripheral peptidergic fibers of the trigeminal nerve in the olfactory bulb of the rat. J Comp Neurol 334:117-124.

Funakoshi K, Nakano M, Atobe Y, Goris RC, Kadota T, Yazama F (2006) Differential development of TRPV1-expressing sensory nerves in peripheral organs. Cell Tissue Res 323:27-41.

Hjerling-Leffler J, Alqatari M, Ernfors P, Koltzenburg M (2007) Emergence of functional sensory subtypes as defined by transient receptor potential channel expression. J Neurosci 27:2435-2443.

Jasmin L, Burkey AR, Card JP, Basbaum AI (1997) Transneuronal labeling of a nociceptive pathway, the spino-(trigemino-)parabrachio-amygdaloid, in the rat. J Neurosci 17:3751-3765.

Ji RR, Samad TA, Jin SX, Schmoll R, Woolf CJ (2002) p38 MAPK activation by NGF in primary sensory neurons after inflammation increases TRPV 1 levels and maintains heat hyperalgesia. Neuron 36:57-68.

Michael GJ, Priestley JV (1999) Differential expression of the mRNA for the vanilloid receptor subtype 1 in cells of the adult rat dorsal root and nodose ganglia and its downregulation by axotomy. J Neurosci 19:1844-1854.

Mishra SK, Hoon MA (2010) Ablation of TrpV1 neurons reveals their selective role in thermal pain sensation. Mol Cell Neurosci 43:157-163.

Mishra SK, Tisel SM, Orestes P, Bhangoo SK, Hoon MA (2011) TRPV1lineage neurons are required for thermal sensation. EMBO J 30:582-593.

Molliver DC, Wright DE, Leitner ML, Parsadanian AS, Doster K, Wen D, Yan Q, Snider WD (1997) IB4-binding DRG neurons switch from NGF to GDNF dependence in early postnatal life. Neuron 19:849-861.

Murata Y, Masuko S (2006) Peripheral and central distribution of TRPV1, substance P and CGRP of rat corneal neurons. Brain Res 1085:87-94.

Neumann S, Braz JM, Skinner K, Llewellyn-Smith IJ, Basbaum AI (2008) Innocuous, not noxious, input activates PKCgamma interneurons of the spinal dorsal horn via myelinated afferent fibers. J Neurosci 28: 7936-7944.

Ruda MA, Ling QD, Hohmann AG, Peng YB, Tachibana T (2000) Altered nociceptive neuronal circuits after neonatal peripheral inflammation. Science 289:628-631.

Sándor K, Helyes Z, Elekes K, Szolcsányi J (2009) Involvement of capsaicinsensitive afferents and the Transient Receptor Potential Vanilloid 1 Receptor in xylene-induced nocifensive behaviour and inflammation in the mouse. Neurosci Lett 451:204-207.

Soriano P (1999) Generalized lacZ expression with the ROSA26 Cre reporter strain. Nat Genet 21:70-71.

Srinivas S, Watanabe T, Lin CS, William CM, Tanabe Y, Jessell TM, Costantini F (2001) Cre reporter strains produced by targeted insertion of EYFP and ECFP into the ROSA26 locus. BMC Dev Biol 1:4.

Tamura S, Morikawa Y, Senba E (2005) TRPV2, a capsaicin receptor homologue, is expressed predominantly in the neurotrophin-3-dependent subpopulation of primary sensory neurons. Neuroscience 130:223-228.

Tominaga M, Caterina MJ, Malmberg AB, Rosen TA, Gilbert H, Skinner K, Raumann BE, Basbaum AI, Julius D (1998) The cloned capsaicin receptor integrates multiple pain-producing stimuli. Neuron 21:531-543.

Willis WD, Westlund KN (1997) Neuroanatomy of the pain system and of the pathways that modulate pain. J Clin Neurophysiol 14:2-31.

Zwick M, Davis BM, Woodbury CJ, Burkett JN, Koerber HR, Simpson JF, Albers KM (2002) Glial cell line-derived neurotrophic factor is a survival factor for isolectin B4-positive, but not vanilloid receptor 1-positive, neurons in the mouse. J Neurosci 22:4057-4065.

Zylka MJ, Rice FL, Anderson DJ (2005) Topographically distinct epidermal nociceptive circuits revealed by axonal tracers targeted to Mrgprd. Neuron 45:17-25. 